\title{
Editorial
}

\section{Special Issue on Recent Advances in Environmental Health Research: Health Disparities, Toxicology and Carcinogenesis}

\author{
Paul B. Tchounwou ${ }^{1}$, and Hongtao $\mathrm{Yu}^{2}$ \\ ${ }^{1}$ Molecular Toxicology Research Laboratory; ${ }^{2}$ Chemical Toxicology/Biochemistry Laboratory, NIH- \\ Center for Environmental Health, School of Science and Technology, Jackson State University, \\ Jackson, Mississippi 39217, USA. Email: paul.b.tchounwou@jsums.edu
}

Received: 21 September 2002 / Published: 30 September 2002

Recent development in biomedical research and clinical sciences has lead to a significant decrease in disease morbidity and mortality, as well as the improvement of quality of life in the United States and around the world. Despite the good news about this improvement in global health status, significant disparities or differences in disease incidence, mental illness, morbidity and mortality continue to exist between specific population groups. The most striking health discrepancies result in shorter life expectancy, as well as higher rates of most cancers, some birth defects, infant mortality, asthma, diabetes, behavioral and affective disorders, cardiovascular, and cerebrovascular diseases in minority groups. Included in these high-risk groups are people with low incomes, low literacy rates, the elderly, those in rural communities, African Americans, Hispanics, American Indians, and Alaska Natives, and other ethnic minorities.

Although poor access to health care, genetic, cultural, behavioral, and socio-economic factors have been linked to health disparities, various environmental determinants including physical, chemical, and biological factors have also been shown to play a key role in disease development and health disparity. Exposure to toxic environmental and occupational agents can have different effects in different persons of differing age, socioeconomic status, ethnic background, gender and genetic composition. Many diseases have been linked to environmental factors, and their effects appear to be different on different population groups due to the multigenic nature of risk factors. Consequently, one of the major challenges in biomedical research today and in the future is to be able to understand the cellular and molecular mechanisms by which various environmental agents interact with other intrinsic and extrinsic factors to induce morbidity and mortality in different population groups. Equally important is the development of computational models to study the physico-chemical properties of these environmental agents and to predict their toxicological effects in various biologic systems.

Under the support of the National Center for Research Resources (NCRR) of the National Institutes of Health (NIH), 23 Research Centers in Minority Institutions (RCMI) have been established among the nation's minority serving institutions. Each year, a selected RCMI institution and NCRR 
co-host an annual meeting to exchange research findings in one or more of the areas concerning health and health disparities. The RCMI 2002 Spring Symposium was held in Jackson, Mississippi in April 24-27, 2002. The theme of the Symposium was "Recent Advances in Environmental Health Research: Health Disparities, Toxicology and Carcinogenesis". The selection of this theme was based on the fact that Health Disparities and Environmental Toxicology/Carcinogenesis are two important research areas of interest to the National Institutes of Environmental Health Sciences (NIEHS) and the NIH. In addition, several RCMI program investigators have in recent years been involved in significant aspects of these research endeavors. The 2002 Spring Symposium covered several important biomedical research topics including the following:

1. Biomarkers and Signal Transduction in Cancer: Recent advances in the molecular biology of the cell cycle regulation have given new life to our understanding of carcinogenesis, and the idea that defects of regulation in cancer cells may partially explain successes that have been achieved in cancer chemotherapy. The areas of research presentation related to this subject included the following: a) biomarkers of effect, sensitivity and effect associated with tumorigenesis; b) molecular mechanisms of carcinogenesis; c) signal transduction and gene activation; d) role of cytokines in tumor biology; and e) molecular targets of cancer chemotherapy.

2. Health Risk Assessment and Management of Environmental Contaminants: Growing public awareness of the potential risk to humans from toxic chemicals in the environment has generated demand for new and improved methods for toxicity assessment and rational means for estimating health risk. The specific areas of research presentation related to this subject included the following: a) hazard assessment of exposure to physical, chemical and biological agents; b) dose-response evaluation and model development; c) exposure assessment analysis; d) health risk characterization; and e) management strategies in health risk prevention and control.

3. New Frontiers in Genetic Toxicology: In recent years a new science termed Toxicogenomics has emerged, with the aim of understanding molecular mechanisms of health and disease, by combining chemical, genomic, and proteomic knowledge into a common framework of the biochemical and genetic pathways to disease. Although this constitutes a new field of research, several symposium presentations dealt with gene expression studies, proteomics, gene-environment interactions, and functional genomics.

4. Emerging Topics in Computational Biology: Using of computational methods and procedures to investigate biological phenomena has made remarkable progresses. This field includes analysis of human genome data, prediction of DNA and protein structure and function, design of biomaterials and therapeutic agents, studies into small molecule-biomacromolecule interactions, and other related computational method development. Therefore, several symposium presentations touched on the computational analysis of the physical and chemical properties of several environmental compounds, as well as on quantitative structure activity relationship (QSAR) studies for developing predictive toxicology models associated with exposure to these compounds. 
5. Environmental Toxicology and Carcinogenesis: There are many environmental agents such as metal ions, polycyclic aromatic hydrocarbons, pesticides/herbicides, UV-light, food additives, and viruses that are known to induce various types of cancer in humans. Several symposium presentations dealt with research elucidating the cellular and molecular mechanisms by which these environmental agents induce toxicity, mutagenesis, and carcinogenesis.

6. Health Disparities and Diseases: With recent advances in laboratory technologies, it is often possible to measure specific genetic variations as risk factors for specific types of cancer. Equally important is the evaluation of the role of modifier factors such as environmental exposures or other genes that may exacerbate the genetic risk leading to differences in cancer susceptibility among individuals. The specific areas of research presentations related to the subject of health disparities included the following: a) health disparities and cancer; b) health disparities and heart disease; and c) health disparities and infectious diseases.

The meeting attracted more than 200 scientists across the disciplines of environmental health and biomedical sciences with more than 125 contributed papers. As stated above, the symposium had six different sessions with more than 25 invited speakers, and two poster sessions. The submitted manuscripts were peer-reviewed and selected for publication by experts in respective fields. The accepted research papers or review articles are being published in four volumes as a special issue of the International Journal of Molecular Sciences. All articles or review articles are related to environmental toxicology, carcinogenesis, and/or health disparities.

We wish to extend special thanks to Dr. Sidney McNairy, Director of Research infrastructure and Research Centers in Minority Institutions at the NIH for his vision and commitment to providing resources for the support of the RCMI centers. Thanks are also extended to all our conference speakers, and especially to Dr. Kenneth Olden, Director of the National Institute of Environmental Health Sciences for giving a distinguished lecture on the important subject of gene-environment interactions. We are also grateful to Dr. Felix Okojie (symposium chair), Dr. William White (chair of the organizing committee), and all members of the planning and implementation committees for their significant contributions to the successful organization of the conference.

Special thanks are extended to Dr. Ronald Mason and Dr. Abdul K. Mohamed, principal investigator and program director respectively, of the RCMI-Center for Environmental Health of Jackson State University. We would like to acknowledge the authors for their involvement and cooperation, and for their outstanding contributions to biomedical research and education. These authors have taken quality time to prepare and revise their manuscripts. Special thanks are also extended to a significant number of peer-reviewers who took time off their busy schedules to carefully and critically review each of the manuscripts.

(C) 2002 by MDPI (http://www.mdpi.org), Basel, Switzerland. 